\title{
Extension of Musculocutaneous Flap Reach Using a Perforator-Pedicled Propeller Flap Technique: A Case Report
}

\author{
Tomoaki Kuroki, MD, PhD ${ }^{1}$ Masakazu Hasegawa, MD, PhD ${ }^{2}$ Noriko Miki, MD² Ugur Horoz, MD ${ }^{3}$ \\ Yasuyoshi Tosa, MD, $\mathrm{PhD}^{1}$
}

${ }^{1}$ Department of Plastic and Reconstructive Surgery, Showa University School of Medicine, Tokyo, Japan

2 Department of Plastic and Aesthetic Surgery, Chiba University, Chiba, Japan

${ }^{3}$ Department of Plastic and Aesthetic Surgery, Ankara Diskapi Yildirim Beyazit Training and Research Hospital, Ankara, Turkey

J Reconstr Microsurg Open 2017;2:e4-e6.

Locating appropriate recipient vessels for anastomosis of flap vessels near lesions can be challenging during the reconstruction of radiation-damaged tissues using free flaps, and the vascular pedicle length of the flap can be insufficient. However, under these conditions, flap reach can be extended by completely dividing it from the main flap using a perforatorpedicled propeller (PPP) flap technique. Furthermore, this technique can be used to optimize flap configurations, such as the shape and direction of the transplant.

In the present case, a thoracodorsal artery perforator (TAP) flap was harvested from within a free latissimus dorsi musculocutaneous (LDMC) flap, and a nuchal radiation ulcer defect was successfully reconstructed without complication. Herein, we report the clinical and surgical details of this case.

\section{Case Report}

A 54-year-old man was admitted to our department complaining of an intractable nuchal ulcer in May 2006. He had received radiotherapy (60 Gy in total) and had undergone surgery for a nuchal squamous cell carcinoma at another hospital 11 years ago. About 10 years after radiotherapy, an ulcer appeared and grew with time. The patient complained of severe nuchal pain and stiffness, and an insufferable smell. Computed tomography analyses showed partial necrosis on the trapezius muscle and on spinous processes of the cervical spine. Tissue debris was removed during the first stage of the operation in June 2006, and 4 weeks after debridement a deep, oval, bowl-shaped soft tissue defect of approximately $13 \times 8 \mathrm{~cm}$ remained. Subsequently, the cervical spine was
Address for correspondence Tomoaki Kuroki, MD, PhD, Department of Plastic and Reconstructive Surgery, Showa University School of Medicine, 1-5-8, Hatanodai, Shinagawa, Tokyo, Japan (e-mail: kurokit@fancy.ocn.ne.jp; keisei@med.showa-u.ac.jp).

exposed at its base, revealing a bowl-shaped defect comprising shallow occipital and deep nuchal parts (-Fig. 1).

\section{Surgical Procedure}

Surgery was designed to reconstruct the oval- and bowl-shaped soft tissue defect on the neck using a free LDMC flap. The carotid trigone was initially dissected to obtain suitable recipient vessels, but extensive radiation-induced fibrosis was observed, and the transverse cervical artery and external jugular vein in the supraclavicular fossa were chosen as recipient's vessels. Because the transverse cervical artery is relatively distal from nape and occipital regions, vertical placement of the LDMC flap at the longitudinal oval defect would require addition of a vein graft. Therefore, a division of the free LDMC flap into two parts to obtain a thick free LDMC flap and a thin TAP flap was considered to optimize the long axis and the thickness of the transplant for oval and uneven tissue defects (-Fig. 2).

The free LDMC flap was then harvested, and vascular anastomosis was performed to cover the deep nuchal defect. The TAP flap was then divided into two perforators as a secondary flap from the distal half of the free LDMC flap, and was rotated approximately 90 degrees clockwise using the PPP flap technique to cover the shallow occipital defect ( - Fig. 3, upper and lower center). Consequently, the long axes of the transplants were adjusted to fit that of the oval tissue defect ( - Fig. 3, right). Accordingly, the deep nuchal defect was filled with the thick LDMC flap and the shallow occipital defect was covered with the thin TAP flap simultaneously in a one-stage procedure without lengthening the vascular pedicle with a vein graft. received

August 25, 2016

accepted

September 8, 2016

published online

October 25, 2016
DOI http://dx.doi.org/

10.1055/s-0036-1593751. ISSN 2377-0813.
Copyright (c) 2017 by Thieme Medical

Publishers, Inc., 333 Seventh Avenue, New York, NY 10001, USA. Tel: +1(212) 584-4662.
License terms

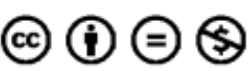



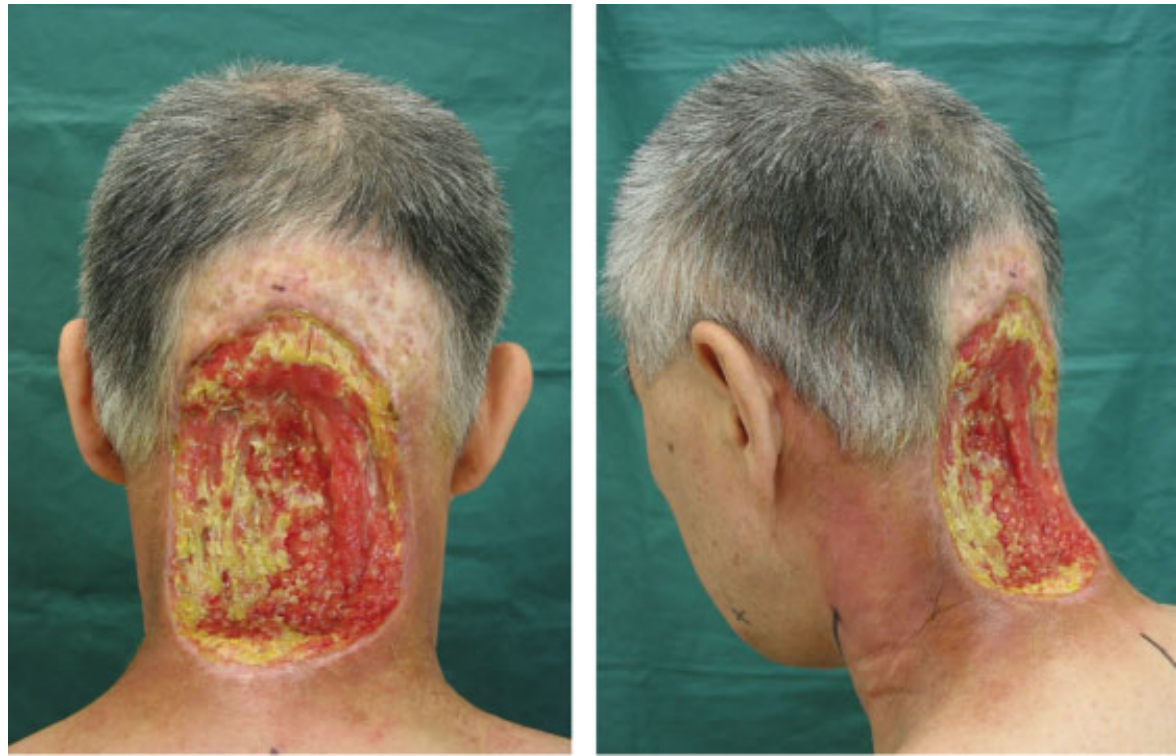

Fig. 1 Preoperative findings. (Left) An oval tissue defect measuring $13 \times 8 \mathrm{~cm}$ remained 4 weeks after debridement. (Right) The defect comprised a shallow occipital part and a deep nuchal part.

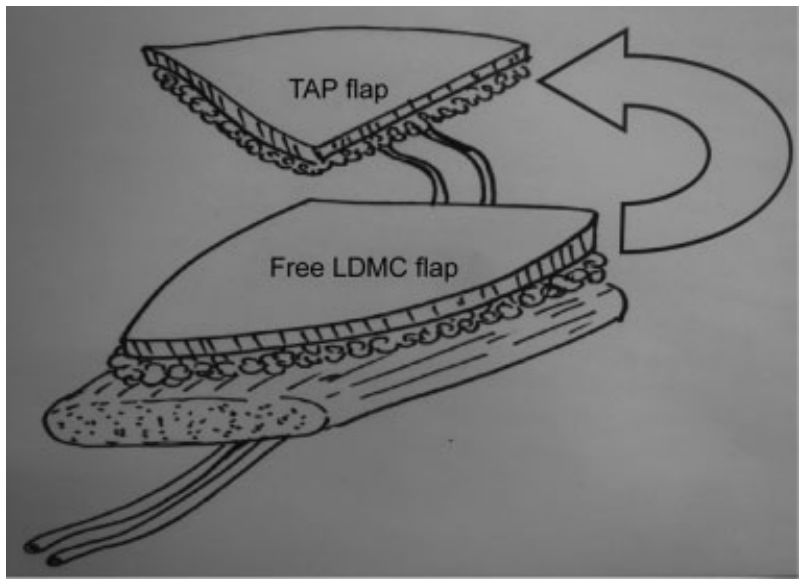

Fig. 2 Schema of the technique. The TAP flap was separated from the distal part of the free LDMC flap. LDMC, latissimus dorsi musculocutaneous; TAP, thoracodorsal artery perforator.

\section{Results}

Both flaps survived without complication, and the nuchal and occipital defects were repaired using a LDMC flap of approximately $12 \times 5.5 \mathrm{~cm}$ and a TAP flap of $10 \times 8 \mathrm{~cm}$ (-Fig. 4).

On the 9-year follow-up visit, the ulcer had not recurred and no pain or restriction due to contracture was noted with motion. Moreover, the transplant retained a good contour without additional defatting.

\section{Discussion}

Flap-transfer using the PPP flap technique is less invasive than that using the conventional flap techniques that were described by Hyakusoku et al in $1991 .{ }^{1}$ Moreover, Jakubietz et al and Wong et al improved this technique with an acentric propeller flap by rotating the flaps by approximately 180
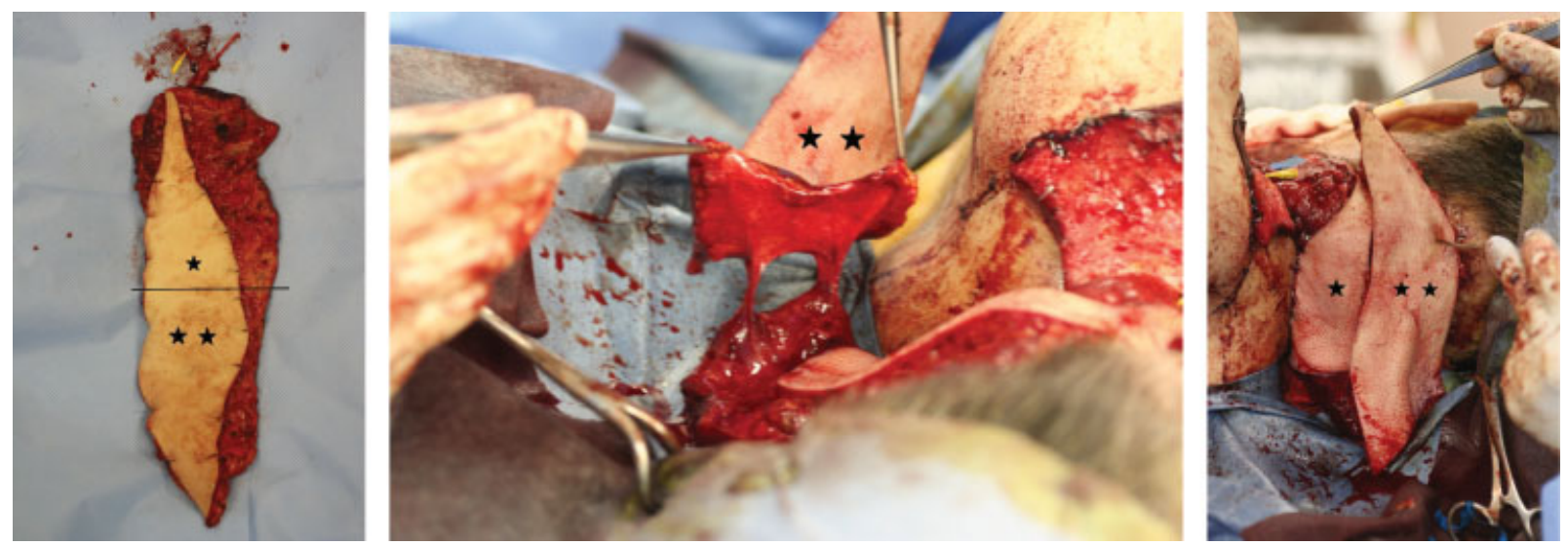

Fig. 3 Harvested free LDMC flap. (Left) The dotted straight line indicates the dividing line. One asterisk indicates the LDMC flap and two asterisks indicate the TAP flap; (center) completion of the TAP flap with the harvest of two perforators; (right) 90 degrees counterclockwise rotation of the divided TAP flap. LDMC, latissimus dorsi musculocutaneous; TAP, thoracodorsal artery perforator. 

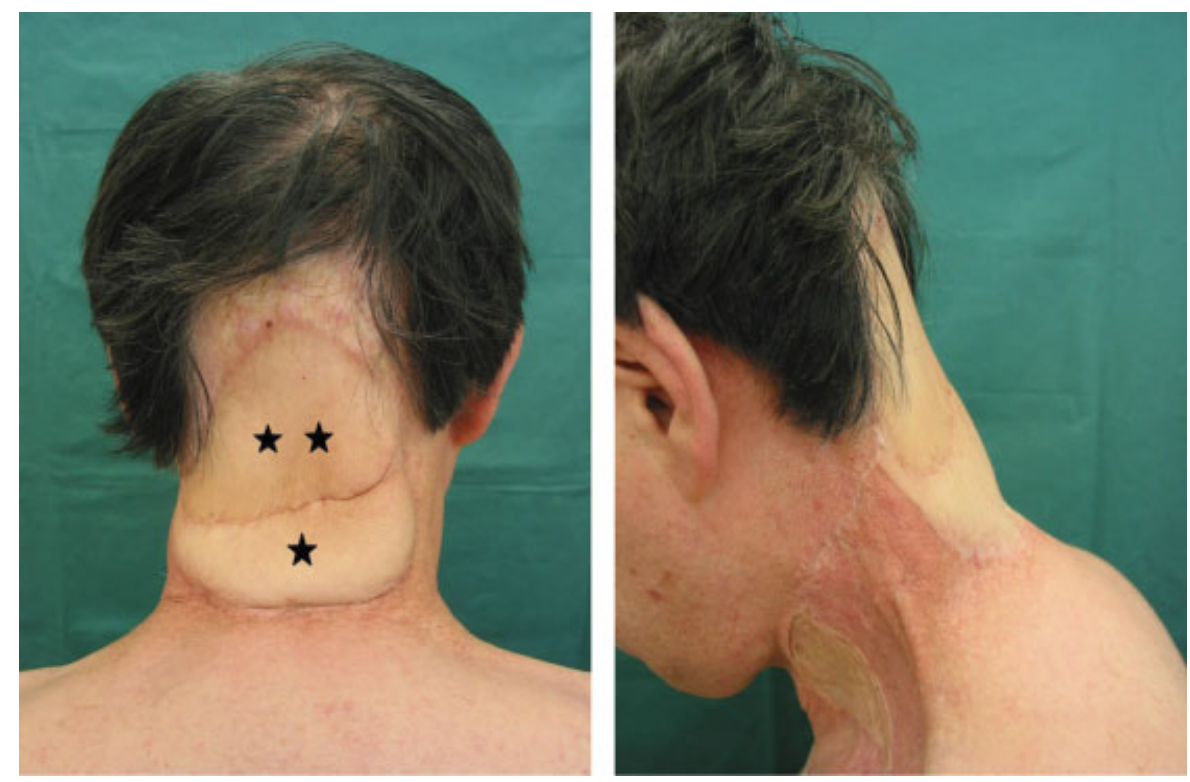

Fig. 4 Appearance of the nape 2 years after surgery. (Left) Nuchal and occipital defects were repaired with an LDMC flap (one asterisk) of $12 \times 5.5 \mathrm{~cm}$ and a TAP flap (two asterisks) of $10 \times 8 \mathrm{~cm}$. (Right) The thick LDMC flap was used to fill the deep nuchal soft tissue defect, and the thin TAP flap was used to cover the shallow occipital defect and gave a natural contour. LDMC, latissimus dorsi musculocutaneous; TAP, thoracodorsal artery perforator.

degrees to cover longer defects with the PPP flaps. ${ }^{2,3}$ We combined this technique with a free LDMC flap to expand the range of reach so that large defects could be reconstructed at a distance from recipient's vessels.

This combined flap could be referred to as a "chimeric flap," and comprises multiple flaps that depend on a single vascular pedicle. ${ }^{4}$ Generally, the purpose of chimeric flaps is to allow other functions of the transplant tissue. Hence, multiple flaps often need to be harvested and vascular anastomosis procedures are required to connect them.

In contrast, the purpose of the present technique was to extend the range of the musculocutaneous (MC) flap, which can reach and optimize the shape or direction of the defect after complete division from the main flap. In addition, this method does not require additional vascular anastomoses and can be applied to various MC flaps for which perforators are available, such as latissimus dorsi, gluteus maximus, vastus lateralis, and rectus abdominis flaps.

Usually, the muscles of MC flaps can be used to fill deep defects and cover dead space. However, in some cases, MC flaps were too bulky and led to cosmetic problems. In contrast, the perforator flap without muscle tissue is suitable for filling shallow lesions. ${ }^{5}$ The present procedure has advantages of both perforator and MC flaps, and the free LDMC flap was used to fill the deep soft tissue defect of the neck, whereas the TAP flap was used to cover the shallow occipital defect (-Fig. 3). Adequate reconstruction of uneven surfaces is difficult with a single flap, and the present novel approach offers an effective solution. To the best of our knowledge, this is the first report to show coverage of nuchal and occipital defects with a TAP flap within a free LDMC flap without lengthening the vascular pedicle. Hence, we recommend the use of this approach to expand the reach of musculocutaneous flaps using a PPP flap technique.

\section{Note}

This article was presented at the 5th Congress of the World Society of Reconstructive Microsurgery (WSRM); 25-27 June, 2009; Okinawa, Japan. The authors have no conflict of interest or financial disclosures. No funding was utilized for the preparation of this article.

\section{References}

1 Hyakusoku H, Ogawa R, Oki K, Ishii N. The perforator pedicled propeller (PPP) flap method: report of two cases. J Nippon Med Sch 2007;74(5):367-371

2 Jakubietz RG, Jakubietz MG, Gruenert JG, Kloss DF. The 180-degree perforator-based propeller flap for soft tissue coverage of the distal, lower extremity: a new method to achieve reliable coverage of the distal lower extremity with a local, fasciocutaneous perforator flap. Ann Plast Surg 2007;59(6):667-671

3 Wong CH, Cui F, Tan BK, et al. Nonlinear finite element simulations to elucidate the determinants of perforator patency in propeller flaps. Ann Plast Surg 2007;59(6):672-678

4 Hallock GG. Simultaneous transposition of anterior thigh muscle and fascia flaps: an introduction to the chimera flap principle. Ann Plast Surg 1991;27(2):126-131

5 Kim JT, Koo BS, Kim SK. The thin latissimus dorsi perforator-based free flap for resurfacing. Plast Reconstr Surg 2001;107(2):374-382 